\title{
¿Son las mujeres más sensibles a los derechos de los animales? Sobre los vínculos entre el animalismo y el feminismo
}

\author{
Are Women More Sensitive to Animal Rights? About the \\ Links between Animal Advocacy Movement and Feminism
}

\author{
IsABel Balza y Francisco Garrido \\ Universidad de Jaén
}

Resumen. En este trabajo se analizan, por una parte, los datos sobre la presencia de las mujeres en los movimientos de derechos de los animales en el estado español; por otro lado, tratamos de encontrar marcos explicativos que aclaren cuál es el vínculo que conecta la sensibilidad feminista con la sensibilidad animalista. Hemos realizado un sondeo sobre el porcentaje de mujeres entre los miembros de las organizaciones animalistas más importantes de España y, con estos resultados, hemos hecho una revisión de los diferentes marcos teóricos que pueden explicar esta significativa orientación de género en la defensa del bienestar animal. Por último, defendemos que hay un vínculo entre la ideología patriarcal y la explotación ecológica, en tanto que ambos comparten la dominación y la opresión de género. En este sentido, la sensibilidad animalista movilizaría sentimientos morales de fraternidad con la naturaleza.

Palabras clave: Animalismo; Feminismo; Ética del cuidado; Ecofeminismo.
AвSTRACT. In this paper we will present on the one hand, the data about women's presence in animal rights movements in Spain; on the other hand, we will try to find explanatory frameworks that clarify which are the link that connects feminist sensibility to animal rights sensibility. We have made a survey on the percentage of women in the membership of the most important animal rights defense organizations in Spain and, with these results, we have made a review of different theoretical frameworks which can explain this significant gender orientation in animal welfare defense. Finally, we claim that there is a bond between patriarchal ideology and ecological exploitation, in the sense that both share domination and gender oppression. In this sense, the sensitivity to animal rights mobilizes moral feelings of fraternity with nature.

Key words: Animal Rights; Feminism; Ethics of Care; Ecofeminism. 


\section{Introducción}

En este trabajo tratamos de responder a la pregunta que da título a este artículo desde dos abordajes distintos: por un lado, desde un enfoque empírico, analizando los datos sobre la presencia de mujeres, dentro del Estado español, en el movimiento animalista de protección de derechos y del bienestar animal. Y por otro lado, desde una perspectiva teórica, tratando de encontrar marcos explicativos que den cuenta de cuál es el vínculo que conecta la sensibilidad feminista con la sensibilidad animalista.

Esta investigación surge a partir de una intuición empírica basada en la experiencia del animalismo español: la presencia muy mayoritaria de mujeres en las asociaciones, plataformas, iniciativas y movimientos de defensa de los derechos de los animales ${ }^{1}$. Para confirmar empíricamente esta intuición, hemos realizado un sondeo sobre el porcentaje de mujeres en los listados de asociados y asociadas de las principales organizaciones animalistas españolas o con fuerte presencia en nuestro país. Estos datos los hemos contrastado con los porcentajes de participación en ONG de las mujeres españolas. Dichos datos están disponibles en las ya abundantes investigaciones publicadas sobre la presencia de mujeres en el mundo asociativo y de las ONG. Dejamos abierta para investigaciones posteriores la cuestión de si la sensibilidad animalista de las mujeres es predicable de todas independientemente de su condición feminista, o lo es especialmente de aquellas mujeres que, de una forma u otra, están en combate contra el patriarcado.
Sobre la base de los datos resultantes hemos llevado a cabo una revisión de los distintos marcos teóricos que podrían explicar esta significativa orientación de género en la defensa del animalismo. En primer lugar, realizamos un repaso histórico sobre los fuertes lazos existentes entre el movimiento sufragista y las primeras organizaciones animalistas, así como de la masiva presencia de mujeres en la fundación de las primeras asociaciones de defensa animal.

Finalmente, sostenemos desde una perspectiva ecofeminista que esta especial sensibilidad animalista va asociada al vínculo inexorable que existe entre patriarcado (en tanto que dominación y explotación de género) y explotación ecológica, en aquella franja (los animales) que está más cerca evolutivamente de nuestra sensibilidad y sistema de comunicación humano. De esta manera, la orientación de género del animalismo nos vendría a mostrar cómo el animalismo es la forma más poderosa de movilización de sentimientos morales de fraternidad con la naturaleza.

\section{Hipótesis y metodología}

Nuestra investigación se fundamenta en dos intuiciones primeras que funcionan a modo de hipótesis de trabajo. Por un lado, una intuición observacional que podemos enunciar de la siguiente manera: "Las mujeres participan en un grado superior a los hombres en las organizaciones animalistas del Estado español". Esta hipótesis se genera a partir de nuestra experiencia, en la que hemos podido observar la masiva presencia de mujeres en los movimientos de defensa de los animales. 
Ello hizo que nos preguntáramos por las razones que pudieran explicar tal dato empírico y, al mismo tiempo, nos hizo plantearnos una segunda cuestión, en este caso, una intuición teórica que enunciamos como sigue: "Las mujeres son más sensibles a los derechos de los animales".

Para evaluar la fortaleza y razón de tales intuiciones hemos medido un indicador de corte claramente cuantitativo, como es la división de género que presenta el voluntariado de las ONG's animalistas del estado español. Y el modo de acceso a tales datos ha sido la utilización del listado de las ONG's animalistas que posee la $\mathrm{APDDA}^{2}$. De tal modo que hemos comprobado los datos referentes a 20 ONG's animalistas y proteccionistas de ámbito internacional, estatal, autonómico y local. Nuestro modo de proceder para acceder a los datos ha sido el de enviar por correo electrónico una carta explicando nuestro objetivo de investigación, junto con un cuestionario en el que se preguntaba sobre los datos de la división de género que presentan los socios y socias voluntarios de la ONG en cuestión ${ }^{3}$.

Dado que en el animalismo confluyen tanto las cuestiones ecológicas ${ }^{4}$, como aquellas actividades vinculadas al cuidado de los otros, hemos comparado los datos recogidos, por una parte, con los datos referidos a otras ONG's ecologistas, como Greenpeace; y, por otra parte, con otras asociaciones cuyo carácter es el de la acción social, como es el caso de la Cruz Roja. Por último, y dado que la tercera cuestión fundamental en nuestra investigación es la del género, también hemos contrastado los datos recopilados con otros estudios y metaestudios sobre voluntariado y género en España. Así, son tres los ejes en los que hemos fijado nuestra observación: el ecologismo, el cuidado de los otros y el género. Todo ello para ver y explicar el modo en el que se cruzan e interrelacionan estas cuestiones en la práctica (y en la teoría).

Así, y tras disponer de la información referida, podemos formular como sigue la hipótesis primaria que habrá de ser contrastada: "Si alguien es mujer tiene una probabilidad mayor de pertenecer a una organización animalista que si es hombre".

Antes de pasar a revisar los datos obtenidos e interpretarlos, detengámonos primero en recordar cuáles son los vínculos históricos entre el feminismo y el animalismo.

\section{Revisión de los lazos entre feminismo $y$ animalismo}

La defensa de los animales no humanos aparece desde el comienzo en la agenda feminista. Como señala Alicia Puleo (2011, 359), feministas pioneras como Olympe de Gouges y Mary Wollstonecraft condenaban la violencia ejercida sobre los animales. Esta mirada hacia la opresión de los otros excluidos - animales no humanos, esclavos y niños-, va a ser una marca que distinga al movimiento feminista ${ }^{5}$. Vamos a repasar aquí brevemente algunos modos en los que los vínculos entre feminismo y animalismo se han dado, así como los argumentos teóricos y las consecuencias que tales alianzas han provocado.

Son varios los momentos en los que la intersección entre el feminismo y el animalismo se ha dado históricamente. 
Por una parte, el animalismo aparece vinculado al feminismo en la génesis misma del movimiento sufragista en la Gran Bretaña de finales del siglo XIX. Y por otra, el animalismo se vincula al movimiento ecofeminista ya desde los años 70 del siglo XX, sea en sus manifestaciones del vegetarianismo, como ya en el ecofeminismo que se formulará dentro de un marco no esencialista. Veamos.

\subsection{Sufragismo y Antiviviseccionismo}

Esta segunda ola del feminismo (según la cronología de los estudios europeos, y primera ola según los estudios norteamericanos) que es el Sufragismo aparece vinculada desde su comienzo con el movimiento animalista. No se trata sólo de que en la fundación de las primeras asociaciones de defensa animal de finales del siglo XIX hubiera una masiva presencia de mujeres ${ }^{6}$, sino que, además, muchas de las mujeres del incipiente movimiento feminista eran animalistas. Este animalismo se traducía en el momento en una lucha contra la vivisección de animales en la experimentación científica (Donovan, 1990). La lista de sufragistas animalistas es larga ${ }^{7}$; así, por ejemplo, Donovan destaca que Margaret Fuller, Emma Goldman y Charlotte Perkins Gilman articulan una crítica del individualismo atómico y del racionalismo en la tradición liberal. Frente a ello proponen una visión que enfatiza la colectividad, la vinculación afectiva y un concepto orgánico de la vida. Para Fuller la liberación de la mujer y su integración en el ámbito público tendría como efecto una feminización de la cultura, lo que supondría el fin de la violen- cia de todo tipo, incluido el sacrificio de animales para comida y la institución del vegetarianismo (Donovan, 1990, 359).

Hay una cuestión que queremos destacar de este primer animalismo feminista que ayuda a comprender las razones del vínculo entre el feminismo y la lucha antiviviseccionista. El viviseccionismo es comparado por Donovan con la sexología de finales del XIX. Esa sería la razón por la que las mujeres sentirían esta afinidad con los animales: por compartir dos modos de dominación que se caracterizan por clasificar a los sujetos (Donovan, 1990, 366). Donovan interpreta el movimiento antiviviseccionista como «una manifestación de una resistencia contrahegemónica emprendida por las mujeres contra la invasión de las nuevas disciplinas ${ }^{8} \gg$ (Donovan, 1990, 367). Del mismo modo que la sexología clasifica en especies y subespecies y anatomiza ("entomologiza", en términos de Foucault) a las mujeres, así se convierten los cuerpos de los animales en máquinas para la disección. Esta es la razón que conduce a la médica Elizabeth Blackwell (1821-1910) a afirmar que la histerectomía y otras cirugías ginecológicas son una extensión de la vivisección (Donovan, 1990, 367). Aquí también debemos mencionar el vínculo entre la vivisección y la pornografía sadomasoquista, en el sentido en que hay obras pornográficas de finales del XIX que incluyen escenas de vivisección (Donovan, 1990, 369).

Pero también encontramos otro argumento que explica la lucha que emprenden las mujeres contra la opresión, bien sea la sufrida por las mujeres o bien sea la sufrida por los animales. En este caso, se trata 
de la idea defendida por algunas sufragistas (y extendida a parte del movimiento feminista posterior) de la presencia de una mayor sensibilidad moral en las mujeres. Así, por ejemplo, la feminista sufragista Frances Power Cobbe (1882-1904) defiende la superioridad moral de las mujeres (González y Rodríguez, 2008, 87-8). Esta idea está implícita en muchas sufragistas que creen tener el deber de acometer la reforma moral de la sociedad.

En definitiva, lo que estas primeras feministas sufragistas y animalistas muestran en sus luchas vinculadas es el necesario lazo que aúna los modos de dominación y opresión, ya se den éstos en forma de sexismo, especismo o esclavismo, e incluso explotación y maltrato infantil. Lo que las mujeres de finales del siglo XIX vieron con claridad es que defender los derechos de un colectivo oprimido, como era el de las mujeres, conllevaba la necesidad de ampliar la reivindicación de esos derechos a los otros dominados como los animales, los esclavos y los niños?.

\subsection{Feminismo cultural y ecofeminismo}

Un siglo más tarde, a partir de la década de los años 70 del siglo XX, volvemos a encontrar una nueva alianza entre el feminismo y el animalismo. Esta vez la defensa de los animales parte desde el ecofeminismo, ya sea en su vertiente vegetariana más radical o en su última versión, crítica con el esencialismo del feminismo cultural. La representante y primera teórica del feminismo vegetariano animalista es Carol Adams, con su libro The Sexual Politics of Meat: A
Feminist-Vegetarian Critical Theory, publicado en 1990.

La defensa del vegetarianismo de Adams se inscribe dentro de lo que se ha venido en llamar feminismo cultural (o feminismo de la diferencia en Europa). Esta corriente teórica del feminismo surge a finales de la década de los años 70 , como una corriente radical y crítica con el feminismo liberal, inscrita dentro de la segunda ola del feminismo (o según la genealogía europea, tercera ola). Lo que el feminismo cultural va a defender es que la dominación de la naturaleza es característica de Occidente y de la psicología masculina, y es la causa del maltrato animal así como de la explotación de las mujeres y del medio ambiente. Frente a ello, se postula un nuevo modo de relación: se subraya el continuum de la naturaleza, frente a la discontinuidad entre especies animales o entre el mundo animal y vegetal, postura defendida por un pensamiento masculino y propiamente occidental. Ahora las formas híbridas son posibles.

En este sentido, Josephine Donovan entiende que la teoría de los animales que denomina masculina (cuyos máximos exponentes serían Peter Singer y Tom Regan) mantiene la jerarquía entre ámbitos naturales y entre especies. En su artículo de 1990, «Animal Rights and Feminist Theory», critica el animalismo fundamentado en el utilitarismo (Singer) y en los derechos naturales o kantismo (Regan). Entiende que es masculino y que se fundamenta en las dicotomías que desde el feminismo se pretenden evitar o trascender (humano/ animal, razón/emoción), y es expresión por ello de un modelo científico matemático 
propio de la Ilustración, que se caracteriza por suprimir el conocimiento basado en las emociones (Donovan, 1990, 365). Esta denominada psicología de la dominación, propia de un modelo de pensamiento masculino y matemático, se caracteriza para Donovan por desechar lo anómalo, siendo que lo anómalo incluye a los animales y a las mujeres (Donovan, 1990, 362). El paradigma newtoniano/cartesiano es el ejemplo de modelo masculino que impone la imagen de la máquina no sentiente para toda la realidad, como Descartes hace con los animales. Siendo que la noción de los animales como seres sin sentimientos o robots inconscientes legitimará su uso, sin ningún tipo de control, en la experimentación científica. Frente a ello, como veremos, Donovan defenderá la ética del cuidado como aquélla que recupera los sentimientos o la emoción para la ética y la más apropiada para un animalismo feminista.

Las primeras manifestaciones del ecofeminismo se inscriben dentro de este feminismo cultural crítico con el pensamiento occidental entendido como masculino y jerarquizador, y como recuerda Carol Adams, defienden la conexión entre la dominación de la mujer y la de la naturaleza, subrayando que los animales son parte de esa naturaleza dominada, y que la condición que ambos, animales y mujeres, comparten es que son seres subordinados y sujetos a la autoridad y al control de otros (Adams, 1996, 200). Adams entiende que el origen del animalismo en los EE.UU. son las comunidades feministas-vegetarianas (Adams, 1996, 198). De este modo, y en su expresión más radical, Adams postula y defiende una versión ecofeminista del ve- getarianismo, ahondando en las ideas que el feminismo cultural plantea. Para Adams, la dominación extrema de una sociedad patriarcal hace que los animales (al igual que las mujeres) sean considerados como mera carne y productos de consumo. Su comparación entre el consumo de carne animal y la pornografía insiste en esta idea de una dominación y opresión compartida entre mujeres y animales no humanos. Como consecuencia de ello, Adams defiende la obligación moral del vegetarianismo, lo que ha sido denominado por Plumwood como "vegetarianismo ontológico". Plumwood critica tal modo de vegetarianismo, proponiendo un "vegetarianismo contextual", que vincula la renuncia a comer carne a las condiciones de explotación y maltrato animal de la actual industria de producción ganadera (Plumwood, 2004).

\subsection{Una ética feminista del cuidado para los animales}

En el artículo de 1990 de Donovan que ya hemos citado, la autora va a plantear la necesidad de una forma de ética que dé cuenta de lo silenciado o negado por aquello que se critica: ahora es necesaria una ética que dé cuenta de la emoción.

La ética que Donovan busca para este animalismo feminista es una "ética de la humildad", que debe caracterizarse por ser una actitud que «acepta no sólo el hecho del dolor y la muerte, sino también el hecho de lo independiente y lo incontrolable, desarrollando y aumentando existencias separadas de las vidas que parece preservar» (Donovan, 1990, 373). Se trata, en palabras de Donovan, de un "amor 
atento" 10 , de una ética que se caracteriza por su cuidado, por su actitud respetuosa hacia los sujetos.

En este sentido, Donovan va a reivindicar a Carol Gilligan y su "Ética del cuidado" (1982) como aquella más apropiada para el animalismo feminista, ya que es una ética "femenina", que se caracteriza por ser contextual y narrativa, más que formal y abstracta. Se contrapone así una "moral de la responsabilidad" (femenina según Gilligan y Donovan), propia del animalismo feminista, frente a la "moral de los derechos" (masculina según las autoras), propia de las propuestas animalistas de Singer (2011) y Regan (2006). En este sentido, también Deborah Slicer (1996) critica el animalismo de Singer y Regan, por olvidar la emoción como parte integral del acto moral; y Brian Luke (1995) va a reivindicar la necesidad de una ética no patriarcal para la liberación animal.

Esta ética del cuidado que se propone desde el feminismo cultural (Carol Gilligan, Sara Ruddick, Estella Lauter, Paula Gunn Allen, Rosemary Radford Ruether y Marilyn French) tiene en común lo siguiente: requiere «un respeto fundamental por las formas de vida no humanas, una ética que escucha y acepta la diversidad de las voces del entorno y la validez de sus realidades» (Donovan, 1990, 374) ${ }^{11}$.

Frente a la imprecisión de su propuesta, Donovan argumenta que su propósito es el de indicar caminos en los que nuestro pensamiento sobre las relaciones entre lo humano y lo animal pueda ser reorientado. No obstante, Donovan tiene claro que esta ética feminista y animalista debe rechazar el consumo de carne, la caza, el asesinato de animales vivos para el vestir, los circos, la ganadería industrial, los experimentos psicológicos y el uso de animales para testados de productos de belleza y limpieza. Se trata de comenzar una conversación, dice, emocional y espiritual con las formas de vida no humanas (Donovan, 1990, 375). Deane Curtin insiste en la misma posición teórica que Donovan. Y entiende la ética del cuidado como una ética ecofeminista (Curtin, 1996, 128), contraponiéndola también a una ética de los derechos.

Ahora bien, el feminismo cultural defiende que el pensamiento relacional de las mujeres hace que éstas se sientan más vinculadas con la naturaleza y con otros seres vivos. Además, a partir de los datos que hemos obtenido podríamos pensar que el animalismo es más propio de las mujeres. Pero ello, no obstante, podría hacernos caer en un pensamiento esencialista, que es precisamente de lo que es acusado el feminismo cultural por otros feminismos de corte liberal. Se critica el feminismo de la diferencia por peligroso, ya que colocaría a las mujeres de nuevo en una posición de subordinación, propia del reduccionismo biológico. Por ello la defensa de los animales no humanos ha sido vista por algunos feminismos como arriesgada e inapropiada (González y Rodríguez, 2008, 99). Esta crítica trataría de establecer nuevas dicotomías que coloquen a la mujer del lado de lo "humano", frente a lo natural-animal.

La propia Donovan revisa su postura de 1990 en un artículo de 2006: aquí el cuidado deja de ser maternal para convertirse en la capacidad -propia de hombres y mujeres- de atender al otro. Incide ahora en el aspecto relacional de la ética y en la 
emoción, no exclusiva de las mujeres: estamos ante una escucha emocional. Por lo que ya la ética del cuidado no se cataloga como una ética "femenina", sino como una ética feminista: es decir, como una ética que recoge aquellos aspectos que el feminismo ha reivindicado para las relaciones humanas, pero no considerándolos exclusivos de las mujeres, sino atribuibles y necesarios para cualquier ser humano. Del mismo modo, Gilligan revisa algunos aspectos de sus propuestas de 1982 sobre la ética del cuidado, insistiendo ahora en que es una ética feminista, que no femenina, en tanto que sus valores son democráticos y universales, afirmando cómo «en un contexto patriarcal el cuidado es una ética femenina», pero «en un contexto democrático, el cuidado es una ética humana» (Gilligan, 2013, 50) ${ }^{12}$.

\section{Resultados iniciales}

De las 45 ONG's contactadas, han respondido 20 (el 44,44\%). Pero estas 20 ONG's son bastante representativas del mundo del voluntariado animalista español: más del $70 \%$ de las asociaciones tienen un carácter voluntario; el 55\% son ONG's estatales y el $45 \%$ son locales o autonómicas; entre ellas hay ONG's de 8 Comunidades Autónomas, incluidas entre éstas las Comunidades que presentan mayor peso demográfico, como son Andalucía, Cataluña y Madrid; el 40\% de las entidades son principalmente animalistas y el $60 \%$ restantes son de carácter prioritariamente proteccionista $^{13}$. Aunque entendemos que la muestra es suficientemente representativa y fiable para nuestros propósitos, debe tenerse en cuenta el carácter limitado de la misma.

\begin{tabular}{|l|r|r|}
\hline ONG & MUJERES $\%$ & HOMBRES $\%$ \\
\hline CACMA & 72,6 & 27,4 \\
\hline ASANDA & 71 & 21 \\
\hline SVPAP & 71,68 & 28,32 \\
\hline ATEA & 70 & 30 \\
\hline PROGAT & 83 & 17 \\
\hline SOS SAGUNTO & 87 & 13 \\
\hline SÁLVANOS & 75 & 25 \\
\hline LA XANA & 74 & 26 \\
\hline RIBERCAN & 86 & 14 \\
\hline FELCAN & 82 & 18 \\
\hline SOFÍ - Refugio Escuela & 82,92 & 17,07 \\
\hline ANIMANATURALIS & 76 & 24 \\
\hline ADDA & 74 & 26 \\
\hline APA SOS BILBAO & 97 & 3 \\
\hline D DE VIDA & 63 & 37 \\
\hline ACDA - Asociación Cacereña para la Protección y Defensa & 76,12 & 23,88 \\
\hline Igualdad Animal / Equanimal & 69 & 31 \\
\hline FAADA & 68 & 32 \\
\hline Amigo Fiel & 50 & 50 \\
\hline LIBERA & 67 & 33 \\
\hline
\end{tabular}

Fuente: propia. Datos de septiembre de $2013^{14}$ 
Como se puede comprobar en el cuadro anterior, la distribución del voluntariado de las organizaciones animalistas encuestadas es muy asimétrico a favor de la mujeres; el 77,68\% de las/os voluntarias/os de las ONG's españolas animalistas y/o proteccionistas consultadas son mujeres y sólo el $21,77 \%$ son hombres.

ONG Animalistas

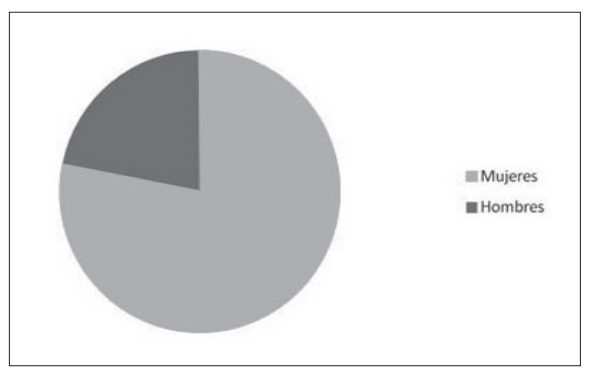

Fuente: Elaboración propia.

Estos datos concuerdan con otras fuentes provenientes de otros estudios sociológicos, donde las mujeres muestran un nivel de sensibilidad mucho mayor hacia el sufrimiento y el maltrato animal, como se ve en el bajo interés que presentan, en especial las mujeres más jóvenes, hacia las corridas de toros.

Interés por las corridas de toros

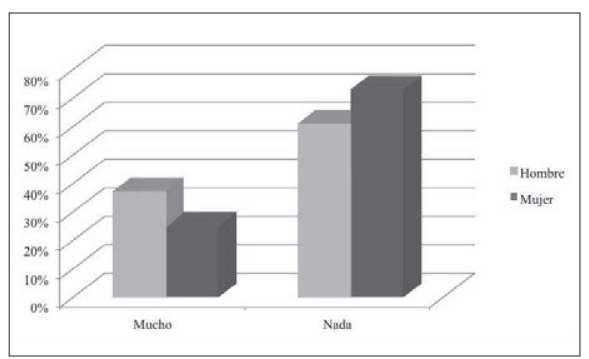

Fuente: Investiga, $2008^{15}$
De modo que estos datos nos ofrecen indicios razonables de que la hipótesis primaria que hemos establecido puede ser apropiada, y podemos formularla como sigue: "Alguien que es mujer tiene una probabilidad 3-4 veces mayor de ser voluntaria/o de una ONG animalista que si es hombre". Y lo que es muy importante: no estamos ante una correlación espuria, sino ante una correlación vigorosa que requiere de una explicación teórica que establezca una relación funcional entre género y animalismo.

\section{El género de las $O N G$}

Para poder entender la singularidad del caso de las asociaciones animalistas en cuanto a la mayor presencia de mujeres entre sus miembros, debemos plantearnos una serie de cuestiones que nos permitan comparar el caso del animalismo con los datos que presentan otras ONG. Así, hemos analizado si la correlación que se da entre género y animalismo se repite en otras ONG y, en el caso de que el sesgo de género fuera evidente, hemos comprobado si son las mujeres las que

Mujeres menores de 24 años

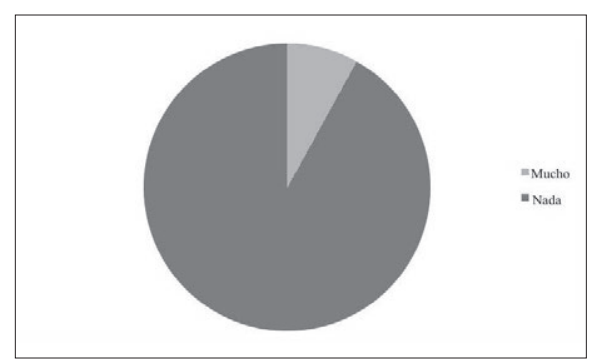

Fuente: Investiga, 2008 
tienen más peso en las asociaciones de carácter voluntario, tal y como ocurre en los grupos animalistas.

Nos hemos fijado en aquellas ONG que presentan algún rasgo en común con las de carácter animalista. Así, nos hemos dirigido a buscar datos de las asociaciones vinculadas por su temática con el animalismo, como son las asociaciones ecologistas o las de acción social, en tanto que son dos caracteres que definen el animalismo.

Efectivamente, una primera aproximación nos permitiría englobar al animalismo dentro del mismo campo semántico del naturalismo ecologista. Aun cuando existen posiciones críticas dentro del animalismo con esta asociación con el ecologismo, aceptaremos que existe cierto aire de familia común. Así, hemos formulado la siguiente pregunta de investigación: “¿En las ONG ecologistas se repite, aunque sea tendencialmente, esta excepcionalidad de género del animalismo?"

Para comprobarlo hemos mirado los datos de una de las ONG ecologistas con mayor número de voluntarios como es Greenpeace $^{16}$. Y nos hemos encontrado con que el número de voluntarios y voluntarias de Greenpeace en España se divide de la siguiente manera: un 45,83\% son mujeres, un $52,60 \%$ son hombres y un $1,27 \%$ de género desconocido. Con lo que podemos concluir que no sólo no se repite la excepcionalidad de género en las ONG ecologistas, sino que además las mujeres son aquí minoría, aunque tengan un peso relevante.
Mujeres menores de 24 años

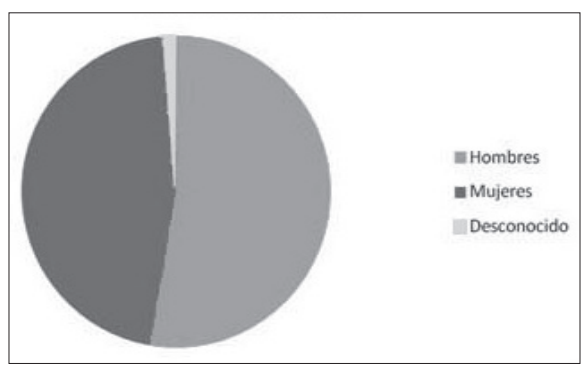

Fuente: Greenpeace España. Datos de septiembre de 2013

Estos datos coinciden también con el nivel medio de sensibilidad hacia los problemas ambientales que muestran los hombres y mujeres. Como se ve, y en lo tocante al género, los resultados son bastante parejos, con una moderada inclinación hacia los hombres, tal como refleja este gráfico extraído de los datos del Barómetro del CIS del 2005.

Interés con el que sigue las noticias relacionadas con los problemas del medio ambiente

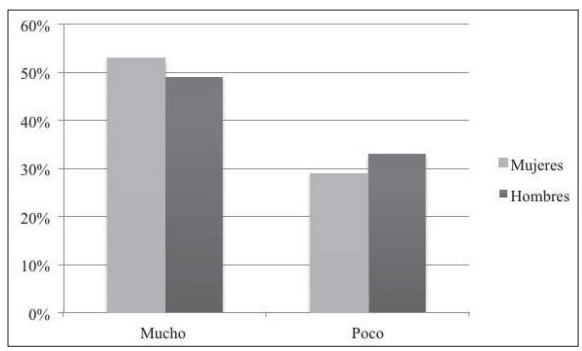

Fuente: Encuesta del CIS 2590, 2005

En las encuestas del CIS sobre medio ambiente, vemos que el interés por el mismo presenta en los datos una correlación de género similar, tal y como nos hemos encontrado en los datos sobre la participación de voluntarios y voluntarias en Greenpeace. 
¿Cree usted que al problema del calentamiento del planeta se le está dando...?

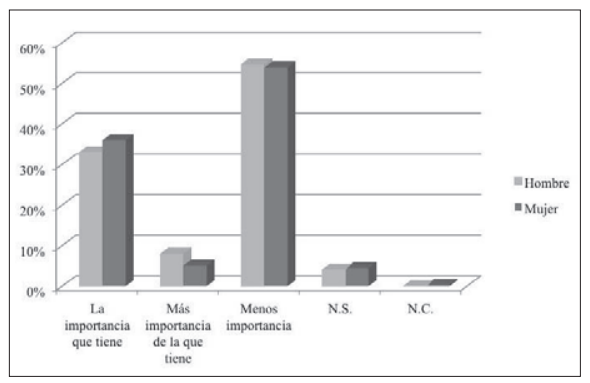

Fuente: Barómetro. CIS 2682. 2007

El otro tipo de ONG en el que hemos recabado los datos del sesgo de género entre sus voluntarios y voluntarias es aquel cuyo objetivo es el de la acción social. En este caso, sus datos nos parecen pertinentes para realizar una comparación con los datos de los grupos animalistas porque las ONG de acción social dedican su actividad de voluntariado a la ayuda, al cuidado o al apoyo de personas y colectivos con algún tipo de desventaja. Y, en este sentido, las ONG de acción social están más orientadas a la ética del cuidado que a la de los derechos, del mismo modo que lo están las asociaciones animalistas.

¿Qué porcentaje de mujeres y hombres hay en las ONG de acción social? Tenemos los datos de la Cruz Roja española ${ }^{17}$, de marcado carácter de acción social, que quizá sea el colectivo de voluntarios y voluntarias más importante por su número y antigüedad que hay en España. Pues bien, el 51\% del voluntariado son mujeres y el $49 \%$ son hombres. Con lo cual vemos que hay una simetría casi perfecta entre este porcentaje y la distribución porcentual por géneros que se da en la sociedad española.

\section{Primeras conclusiones}

La presencia de las mujeres en las $\mathrm{ONG}$ animalistas es muy mayoritaria en unos porcentajes que no tienen parangón con ningún otro ámbito del voluntariado $\mathrm{y}$, en este sentido, constituye un correlato excepcional.

Esta correlación tan fuerte entre género y voluntariado animalista no tiene equivalente en ningún otro tipo de $\mathrm{ONG}$ asimilable, como son las de carácter ecologista o de acción social.

Las ONG de acción social sí comparten la tendencia de las ONG animalistas, con un peso mayoritario de mujeres, no así las ONG ecologistas donde el peso de las mujeres es minoritario ${ }^{18}$.

Estos datos podrían hacer pensar que las ONG animalistas comparten con las ecologistas gran parte de la temática objeto (solidaridad interespecífica y obligaciones extraespecíficas), pero no las formas de acción, orientación y motivación (ética del cuidado) de las conductas; mientras que, por el contrario, comparten las formas de acción, orientación y motivación de las conductas con las ONG de acción social, pero no su temática objeto.

A pesar de la excepcional correlación de género en el voluntariado animalista, lo cierto es que el número de mujeres españolas que participan activamente o simpatizan con la causa animalista es muy bajo en comparación con las que participan en el voluntariado de ONG de acción social e incluso ecologistas.

Además, la distribución de género es homogénea en las ONG de acción social, con tendencia hacia un mayor peso de las 
mujeres; mientras que en las ONG ecologistas se da la tendencia inversa, con un mayor peso de los hombres, pero esto se rompe en las ONG animalistas.

Señalar, por último, que quizás la pregunta no sea por qué participan tantas mujeres en las ONG animalistas, sino por qué participan tan pocos hombres.

\begin{tabular}{lcc}
\hline & Mujeres & Hombres \\
\hline $\begin{array}{l}\text { ONG } \\
\text { Animalistas }\end{array}$ & $74.76 \%$ & $24.83 \%$ \\
\hline $\begin{array}{l}\text { ONG } \\
\text { Ecologistas }\end{array}$ & $45.83 \%$ & $52.90 \%$ \\
\hline $\begin{array}{l}\text { ONG } \\
\text { Cruz Roja }\end{array}$ & $51 \%$ & $49 \%$ \\
\hline $\begin{array}{l}\text { ONG } \\
\text { Acción } \\
\text { Social }\end{array}$ & $56.40 \%$ & $43.60 \%$ \\
\hline
\end{tabular}

Fuente: Elaboración propia

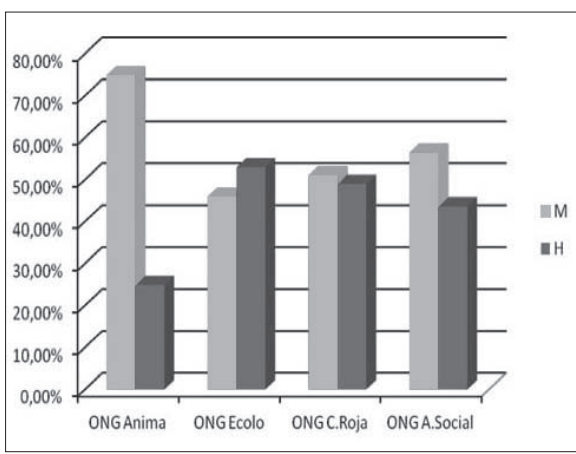

Fuente: Elaboración propia

\section{Hipótesis explicativas}

Hemos formulado tres hipótesis que expliquen por qué dentro de las agrupaciones animalistas se da este mayor peso de la presencia de las mujeres; recordemos los datos: un $77,68 \%$ de las/os voluntarias/os de las ONG españolas animalistas consultadas son mujeres y el $21,77 \%$ restante son hombres. Estas son, pues, nuestras hipótesis:

En primer lugar, y como primera hipótesis, observamos que, ya desde su comienzo, en el movimiento animalista se aúnan tres tendencias de las mujeres:

a) La reivindicación de la ampliación de los derechos a todas y todos las dominadas y dominados por el heteropatriarcado, lo que podría señalarse como esa "pasión igualitaria" del sufragismo; característica, por su parte, del feminismo de la igualdad o liberal. b) El animal es contemplado como el representante directo con vínculos emocionales más cercano dentro de la naturaleza. En este sentido, se postula que las mujeres tendrían menos fracturada la unidad primordial entre naturaleza y sociedad, o entre cognición y emoción; tesis defendida por el feminismo cultural norteamericano y por los primeros ecofeminismos.

c) El animal se define como sujeto y objeto dentro del universo de la ética del cuidado y de la compasión; ética que también se postula como la más pertinente para el feminismo, según el feminismo cultural y/o de la diferencia.

En segundo lugar, y como segunda hipótesis, planteamos que el animal ha sido percibido por parte de las mujeres como, lo que podríamos llamar, compañero de la celda doméstica. Teniendo en cuenta lo afirmado más arriba, en cuanto a los modos de opresión heteropatriarcal compartidos por animales no humanos y mujeres, 
se habría dado una especie de alianza en la dominación, contemplando las mujeres a los animales no humanos y, en concreto, a los animales domésticos, como aliados y compañeros dentro de la estructura de dominación heteropatriarcal.

Por último, y como tercera hipótesis explicativa, entendemos que el modelo masculino de socialización (muy logocéntrico y raciocéntrico) mantiene un fuerte bloqueo a la empatía interespecífica. Ello no significa que los hombres no puedan participar de esta empatía hacia los animales no humanos, pues no se trata de una hipótesis que defienda el esencialismo de los comportamientos de los hombres y de las mujeres. Lo que defendemos es que culturalmente son las mujeres en su mayoría las que han desarrollado la empatía hacia los animales no humanos $\mathrm{y}$, en consecuencia, los modelos educativos han asimilado esos hábitos a lo femenino, con lo que el acceso a tales hábitos ha sido vedado o dificultado para los hombres.

\section{Lagunas y deficiencias del programa de investigación y nuevos protocolos}

Los resultados que hemos obtenido en esta investigación son alentadores en el sentido en que corroboran nuestra primera hipótesis nacida de la intuición; a saber: "Las mujeres son más sensibles a los derechos de los animales", al mismo tiempo que hemos formulado argumentos que, desde la teoría feminista y desde el ecologismo, dan cuenta de ello.

No obstante, el estudio carece de datos sobre componentes demográficos relevantes como la clase, la edad, la for- mación o el territorio. Por otra parte, la distinción entre ONG animalista y ONG proteccionista no está suficientemente discriminada. Además, no hemos introducido datos globales demográficos sobre la opinión de mujeres y hombres ante el maltrato animal y los derechos de los animales, ni tenemos datos sobre la mayor o menor empatía de hombres y mujeres ante el sufrimiento animal.

Por ello, nos planteamos realizar nuevos protocolos que continúen con esta investigación, que incluirían las siguientes acciones: realizar una encuesta con muestreo aleatorio entre voluntarias de ONG animalistas sobre conductas, opiniones y actitudes donde queden reflejadas las variables demográficas aludidas; diferenciar entre ONG animalistas y proteccionistas; analizar estudios de opinión genéricos sobre maltrato animal, ecología o derechos sociales en función del género; realizar un estudio cualitativo con historias de vida y entrevistas semiestructuradas con voluntarias de ONG animalistas sobre la cuestión feminista; y diseñar un ensayo experimental donde medir la mayor o menor empatía de hombres y mujeres ante el sufrimiento animal.

Para acabar, queremos esbozar una de las preguntas que nos hemos planteado a lo largo de este trabajo y que dejamos para una futura investigación: ¿La movilización animalista es intrínsecamente feminista?

Efectivamente, sabemos que son las mujeres las que copan los movimientos animalistas, pero no hemos podido establecer una relación directa entre la participación en las ONG animalistas y la 
conciencia y/o militancia feminista de estas mujeres. La cuestión que debemos plantearnos es pensar si el animalismo es intrínsecamente una cuestión de género o no. En principio no lo es, pero, de hecho, hay muchas más mujeres en los movimientos animalistas, tanto en el Estado español, como en los Estados Unidos (Einwohner, 1999).

En este sentido, en uno de los pocos estudios que hay sobre este tema en el Estado español, Del Valle Moreno, analizando el caso del movimiento animalista en Galicia, concluye que la concienciación de la explotación animal por parte de las mujeres del movimiento animalista no conlleva la conciencia de la explotación de género (Del Valle Moreno, 2004, 299). Es decir, la autora concluye que hay una falta de conciencia feminista entre los miembros del Movimiento animalista en Galicia. Por ello, habría que estudiar si esta falta de conciencia feminista por parte de las mujeres pertenecientes a los movimientos animalistas se repite en otras asociaciones de defensa de los animales no humanos.

\section{Coda: ¿Debe el animalismo formar parte de la agenda feminista?}

¿Puede el movimiento animalista ser un lugar primero de concienciación política que conecte a las mujeres (no feministas) con la conciencia feminista? Y aquí sería pertinente plantearse cómo movilizaciones no feministas, que se conectan con roles y responsabilidades tradicionales de las mujeres (el cuidado o los animales, por ejemplo) "son con frecuencia interesantes laboratorios donde convi- ven formas muy diversas de entender la participación y donde muchas mujeres participan políticamente sin tener necesariamente una experiencia previa de socialización militante" (Alfama i Guillén, 2009, 128). Si bien la influencia del género en la emergencia y desarrollo de todo movimiento social ya ha sido estudiada y no puede ser negada (Taylor, 1999), en nuestro caso se trataría de analizar cómo puede surgir un compromiso feminista a partir de otros compromisos políticos en principio alejados de las luchas del feminismo.

El animalismo es el espacio de acción política donde más confluyen el ecologismo y el feminismo o, dicho de otra manera, el ecologismo feminista se da, mayoritariamente, vinculado a su versión animalista. O al menos surge de ahí, tal y como hemos visto en el caso de los Estados Unidos. Así que podríamos pensar que el animalismo debería ser un buen lugar activista para que la conciencia feminista emergiera.

Otra cuestión sería la de pensar si el animalismo debe formar parte de la agenda feminista: una vez superado el miedo de ciertos feminismos liberales a que las mujeres queden encasilladas o biologizadas/esencializadas en su rol de cuidadoras, el feminismo contemporáneo entiende que tanto el cuidado como la compasión no son exclusivos de las mujeres, sino otros modos de relación que deben ser compartidos y adoptados por cualquier ser humano. Y aquí la cuestión animalista es prioritaria. Queda ello pendiente para un próximo trabajo. 


\section{BIBLIOGRAFÍA}

Adams, Carol J., The Sexual Politics of Meat: A Feminist-Vegetarian Critical Theory, Continuum, New York, 1990.

Adams, Carol J., «Ecofeminismo y el Consumo de Animales», en Warren, Karen J. (ed.), Filosofías ecofeministas, Icaria, Barcelona, 1996, pp. 195225.

Alfama i Guillén, Eva, «Hacia la perspectiva de género en el estudio de los movimientos sociales. La participación de las mujeres en la Plataforma en Defensa de l'Ebre», REIS, 2009, $\mathrm{n}^{\circ} 125$, pp. 117-130.

CIS, «Ecología y medio ambiente (II)», Estudio 2590, enero 2005, http://www. cis.es/cis/opencms/-Archivos/Boletines/38/BDO 38 informacion.html (consultado el 10-1-2015).

CIS, «Ecología y medio ambiente (III)», Estudio 2682, marzo 2007, http://www. cis.es/cis/export/sites/default/Archivos/ Marginales/2680 2699/2682/Cru268200SEXO.html (consultado el 101-2015)

Curtin, Deane, «Hacia una ética de respeto por la naturaleza», en Warren, Karen J. (ed.), Filosofias ecofeministas, Icaria, Barcelona, 1996, pp. 127-148.

Del Valle Moreno, Alejandra, "Género y movimiento de liberación animal: el caso de Galicia», en Cavana, Puleo y Segura (coords.), Mujeres y Ecología. Historia, Pensamiento, Sociedad, Almudayna, Madrid, 2004.

Derrida, Jacques, El animal que luego estoy si(gui)endo, Trotta, Madrid, 2008.

Donovan, Josephine, «Animal Rights and Feminist Theory», Signs, 15 (2), 1990, pp. 350-375.

Donovan, Josephine, «Feminism and the Treatment of Animals: From Care to Dialogue», Signs, 31 (2), 2006, pp. 305-330.
Einwohner, Rachel L., «Gender, Class, and Social Movement Outcomes. Identity and Effectiveness in Two Animal Rights Campaigns», Gender \& Society, 13 (1), 1999, pp. 56-76.

Gaarder, Emily, Women and the Animal Rights Movement, Rutgers University Press, New Brunswick, New Jersey and London, 2011.

Gilligan, Carol, In a Different Voice: Psychological Theory and Women's Development, Harvard University Press, Cambridge, 1982.

Gilligan, Carol, «La resistencia a la injusticia: una ética feminista del cuidado», en La ética del cuidado, Cuadernos de la Fundació Víctor Grífols i Lucas, 30, Barcelona, 2013.

González, Marta y Rodríguez, Jimena, «Al margen de los márgenes: encuentros y desencuentros entre feminismo y defensa de los animales», en González, Riechmann, Rodríguez Carreño y Tafalla (coords.), Razonar y actuar en defensa de los animales, Los Libros de La Catarata, Madrid, 2008, pp. 83-106.

Investiga, «Interés por las corridas de toros», 2008, http://asanda.org/documentos/tauromaquia/encuestas-sobrecorridas-de-toros/01.08.2008 (consultado el 15-12-2014).

Kemmerer, Lisa (ed.), Sister Species. Woman, Animals and Social Justice, University of Illinois Press, UrbanaChampaign, 2011.

Luke, Brian, «Taming Ourselves or Going Feral? Toward a Nonpatriarchal Metaethic of Animal Liberation», en Adams and Donovan (eds.), Animals and Women: Feminist Theoretical Explorations, Duke University Press, Durham and London, 1995, pp. 290-319.

Plumwood, Val, «Feminismo y ecología: ¿Artemisa versus Gaia?», en Cavana, Puleo y Segura (coords.), Mujeres y 
Ecología. Historia, Pensamiento, Sociedad, Almudayna, Madrid, 2004, pp. 53-105.

Puleo, Alicia H., Ecofeminismo para otro mundo posible, Cátedra, Madrid, 2011.

Regan, Tom, Jaulas vacias: el desafio de los derechos de los animales, Fundación Altarriba, Barcelona, 2006.

Ruddick, Sara, «Maternal Thinking», $\mathrm{Fe}$ minist Studies, 2, 1980.
Singer, Peter, Liberación animal. El clásico definitivo del movimiento animalista, Taurus, Madrid, 2011

Slicer, Deborah, «iTu perro o tu hija?: una reflexión feminista sobre la experimentación animal», en Warren, Karen J. (ed.), Filosofias ecofeministas, Icaria, Barcelona, 1996, pp. 171-193. Taylor, Verta, «Gender and Social Movements: Gender Processes in Women's Self-Help Movements», Gender \& Society, 13:8, 1999, pp. 8-33.

\section{NOTAS}

${ }^{1}$ La presencia mayoritaria de mujeres dentro del movimiento de derechos de los animales la encontramos también en otros países; así, por ejemplo, el trabajo de Gaarder centrado en los Estados Unidos parte asimismo de esta premisa empírica en su análisis (Gaarder, 2011, 1)

${ }^{2}$ Asociación Parlamentaria de Derechos de los Animales. Este censo se encuentra en la base de datos de la APDDA, y se puede consultar en ésta, aunque no es de dominio público por contener datos privados (direcciones, correos electrónicos).

${ }^{3}$ Agradecemos a la Asociación Parlamentaria de Derechos de los Animales y a Anna Puig, su secretaria de comunicación, la ayuda que nos han prestado a la hora de obtener esta información.

${ }^{4}$ Lo que nos hace sostener la convergencia entre animalismo y ecologismo no es meramente un simple análisis conceptual sobre los metadiscursos éticos, sino un análisis empírico de las acciones y de los discursos del activismo animalista y ecologista. Nos inscribimos, así, en el campo de la ética experimental y sostenemos sobre datos empíricos la tesis de la convergencia.

${ }^{5}$ Incluso Derrida, al criticar la visión que de los animales se ha articulado en la filosofía, insinúa que hay una diferente perspectiva de género sobre los animales no humanos por parte de filósofos y filósofas, aunque no llega a analizarla: «...todos ésos y no todas ésas, porque esta diferencia no es aquí insignificante» (Derrida, 2008, 29).

${ }^{6}$ Así lo constata Gaarder, citando a Elston: «Women soon constituted a majority of RSPCA members, growing from 50 percent in 1850 to 69 percent in 1900» (Gaarder, 2011, 7).

${ }^{7}$ Sufragistas animalistas eran: Mary Wollstonescra$\mathrm{ft}$, Harriet Beecher Stowe, Lydia Maria Child, Elizabeth Blackwell, Elizabeth Stuart Phelps Ward, Susan B. Anthony, Victoria Woodhull, Elizabeth Cady Stanton, las hermanas Grimké, Lucy Stone, Frances Willard, Frances Power Cobbe, Anna Kingford, Caroline Earle White y Agnes Ryan.
${ }^{8}$ La traducción del texto de Donovan es nuestra.

${ }^{9}$ Aunque no podemos olvidar la existencia de conflictos entre los derechos de los animales y los derechos de las mujeres. Como señalan González y Rodríguez Carreño: "feminismo y antiviviseccionismo no fueran siempre fácilmente compatibles, pues, en ocasiones, la defensa de los animales chocaba con reivindicaciones feministas tan básicas como el acceso de las mujeres a una educación superior en igualdad de condiciones que los hombres. Frente a las ideas de Cobbe y las antiviviseccionistas, muchas feministas reclamaban el derecho de las mujeres a tener acceso a idénticas oportunidades con los hombres, incluida la misma formación, aunque ello conllevara participar en la realización de vivisecciones" (González y Rodríguez, 2008, 88). 1980.

${ }^{10}$ Citando a Sara Ruddick en «Maternal Thinking»,

${ }^{11}$ Aunque aquí no podemos entrar en ello, queremos señalar también el interés de utilizar los conceptos de performatividad y agencia analizados por Butler y Latour, por la posibilidad que plantean de examinar las prácticas de actores humanos como no humanos y el modo en que se construyen en su interacción la "humanidad" o la "animalidad" (González y Rodríguez, 2008, 102)

${ }^{12}$ También desde el ecofeminismo, Alicia Puleo reivindica para el animalismo una ética del cuidado, pero complementada con una ética de los derechos. Resaltando la pertinencia de una ética relacional para el animalismo, no olvida la necesidad de poder establecer reglas que estén más allá del contexto concreto de cada caso, para lo cual deberemos acudir a una ética de los derechos (Puleo, 2011, 376).

${ }^{13} \mathrm{La}$ diferencia entre organizaciones animalistas y proteccionistas es cada vez más difusa pero podemos establecerla en la distinción entre organizaciones reivindicativas dedicadas al reconocimiento y protección de derechos de los animales (animalistas) y las organizaciones de acción social que tienen como fin el cuidado y protección práctica de animales concretos 
¿Son las mujeres más sensibles a los derechos de los animales? Sobre los vínculos entre el animalismo...

(proteccionismo). Entre ambos modelos hay zonas de intersección, pues los animalistas suelen tener refugios y servicios de cuidado y los proteccionistas están también implicados en campañas de reivindicación de derechos.

${ }^{14}$ Con respecto a los datos de ASANDA, que señalan que la pertenencia es de un $71 \%$ de mujeres y un $21 \%$ de hombres, queremos señalar que la ausencia de adscripción de género del $8 \%$ restante es un vacío en la información suministrada que responde exclusivamente a razones técnicas (olvido, no registro, etc.), y que carecemos de datos para poder realizar una interpretación intencional de dicho vacío.

${ }^{15}$ Dado que la empresa responsable de la encuesta ha cerrado, damos a continuación la ficha técnica del estudio. Ámbito: Nacional excepto Ceuta, Melilla y las Islas Canarias. Universo: Población de 15 y más años de edad. Muestra: 1.013 casos. Entrevistas: Personales en el hogar del entrevistado. Selección: Aleatoria de secciones censales para la determinación del hogar y por cuotas de sexo y edad para el entrevistado. Trabajo de campo: Del 1 al 23 de Agosto de 2008. Margen de error: $\pm 3,14 \%$ para $\mathrm{p}=\mathrm{q}=0,5$ y un nivel de confianza del $95,5 \%$ para datos globales. Instituto responsable: IG-INVESTIGA, S.A.

${ }^{16}$ Información proporcionada por Greenpeace como respuesta a nuestro requerimiento.

Los datos son escasos en cuanto al número de asociaciones utilizadas, pero no en cuanto al número de asociados, pues Greenpeace es con una diferencia muy significativa, posiblemente por encima del $80 \%$, quien reúne la mayoría de asociados ecologistas del estado español. Si tenemos en cuenta que no existen diferencias ideológicas ni programáticas sustantivas entre las distintas grandes asociaciones ecologistas del estado español (Greenpeace, Ecologistas en Acción, WWF/ España y SEO/BirdLife), tomar como representativa la muestra de los asociados de la ONG con mayor afiliación y mejores datos registrales es altamente confiable.

${ }^{17}$ Información proporcionada por la Cruz Roja como respuesta a nuestro requerimiento.

${ }^{18}$ Dado que sólo ofrecemos datos relativos a Greenpeace, queremos aclarar lo siguiente. Hay dos grandes organizaciones ecologistas de ámbito estatal en España: Greenpeace y Ecologistas en Acción. Hay consenso en que Greenpeace es la ONG que tiene un número mayor de socios y socias y una mayor diversidad de extracción social ideológica y territorial. Sólo analizando los ingresos anuales de ambas organizaciones se demarca claramente la dimensión de una y otra, habida cuenta de que Greenpeace no puede recibir otros ingresos que los obtenidos por sus asociados. Greenpeace ofrece datos precisos y rigurosos sobre sus afiliados y afiliadas, cosa esta que no ocurre con Ecologistas en Acción, en el momento de la consulta para este estudio. El tamaño de la muestra que representa la totalidad de la afiliación de Greenpeace con respeto al total de la población ecologista en España satisface con demasía los parámetros de significatividad estadística establecidos para que los datos sean representativos y las conclusiones que se extraigan sean muy robustas. 